\title{
Laser-flash in-plane thermal analysis: the case of oriented UHMWPE
}

\author{
Giuseppe Forte, Sanjay Rastogi, Sara Ronca \\ Department of Materials, Loughborough University, Loughborough, LE11 3TU, UK
}

\begin{abstract}
Laser-flash thermal analysis has been applied to measure the thermal diffusivity of highly oriented samples of Ultra High Molecular Weight Polyethylene. Due to the anisotropy of the sample, in-plane measurements are required instead of through-plane ones.
\end{abstract}

Keywords: Laser-flash thermal analysis, thermal diffusivity, thermal conductivity, oriented UHMWPE. PACS 81

\section{INTRODUCTION}

Processing of Ultra High Molecular Weight Polyethylene needs special techniques due to the high melt viscosity of the material, a consequence of the numerous entanglements existing between chains. By controlled synthesis ${ }^{1}$ we have synthesized a wide range of linear UHMWPEs with reduced number of entanglements that can be stretched uniaxially into tapes.

In these uniaxially drawn tapes the bundles of fibres align themselves in a preferred crystal plane orientation (Figure 1) accounting for extraordinary high tensile modulus/strength. We have reported ${ }^{2}$ values approaching $4.5 \mathrm{GPa}$ for tensile strength and $200 \mathrm{GPa}$ for the modulus of uniaxially stretched tapes of this 'disentangled' UHMWPE.

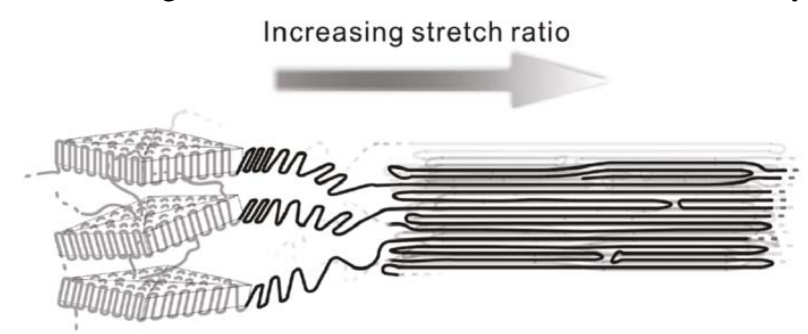

Figure 1. Alignment of polymeric chains as a result of stretching ${ }^{1}$

The stretching process is accomplished in the solid state, with no need for any solvent. The high orientation is not only responsible for the high modulus/strength in tapes, but it has the potential to provide unique physical properties, such as thermal conductivity through lattice vibrations.

One of the best thermal conductors known is a non-metallic material: a natural, single crystal of diamond has a value of thermal conductivity of $2190 \mathrm{~W} / \mathrm{mK}$, due to the very efficient transmission of heat through the lattice vibrations in a perfect crystal. This suggests that in principle (semi) crystalline polymers could also have high thermal conductivities, provided that the lattice vibrations could be transmitted 'unperturbed' along the chains. This condition is not met in bulk polymers (even if semi-crystalline) because of the random size, orientation and distribution of the crystalline domains and the presence of the amorphous component. But what happens if the chains get aligned and packed in larger, well oriented crystalline domains?

The possibility of thermal conductivity in oriented polymers was first reported by Choi et al. in $1978^{3}$ where the authors measured, by using a laser-flash technique, a thermal conductivity of $14 \mathrm{~W} / \mathrm{mK}$ for a sample of polyethylene stretched to a draw ratio of 25: this should be compared with values below $0.5 \mathrm{~W} / \mathrm{mK}$ for non-oriented, bulk PE. More recently, Shen and co-workers ${ }^{4}$ have reported values as high as $104 \mathrm{~W} / \mathrm{mK}$ for highly oriented nanofibres of 
UHMWPE, but, due to the peculiar nature of the sample, a technique based on AFM had to be developed to measure the thermal properties, thus making it difficult to compare with values obtained by typical techniques.

Laser-flash thermal analysis has been successfully applied to measure thermal properties of materials such as thermal diffusivity and thermal conductivity: dos Santos and co-workers have shown that this technique can be suitably used also to assess the thermal properties of polymeric materials ${ }^{5}$.

The technique was introduced for the first time in 1961 by Parker et al. ${ }^{6}$ : short pulses of heat are sent to the front of a specimen, while recording the temperature rise on the back. Using the half time $\left(\mathrm{t}_{1 / 2}\right.$, time value at half signal height) and sample thickness (d), the thermal diffusivity ( $\alpha$, expressed in $\mathrm{m}^{2} / \mathrm{s}$ ) can be measured according to the equation (1):

(1) $\alpha=0.1388 \frac{d^{2}}{t_{1 / 2}}$

From the thermal diffusivity, the thermal conductivity $k$ (in W/m K) can be calculated according to equation (2):

(2) $\alpha=\frac{k}{\rho c_{p}}$

Where $\rho$ is the material bulk density in $\mathrm{kg} / \mathrm{m}^{3}$ and $\mathrm{c}_{\mathrm{p}}$ is the specific heat in $\mathrm{J} / \mathrm{kg} \mathrm{K}$. The method gives a direct measurement of the thermal diffusivity, while the conductivity is obtained indirectly.

The measurements as described above will measure the thermal diffusivity through an ideally isotropic sample: for samples that are anisotropic in nature, a variation of this basic set-up should be applied, namely an in-plane type of measurement (Figure 2).

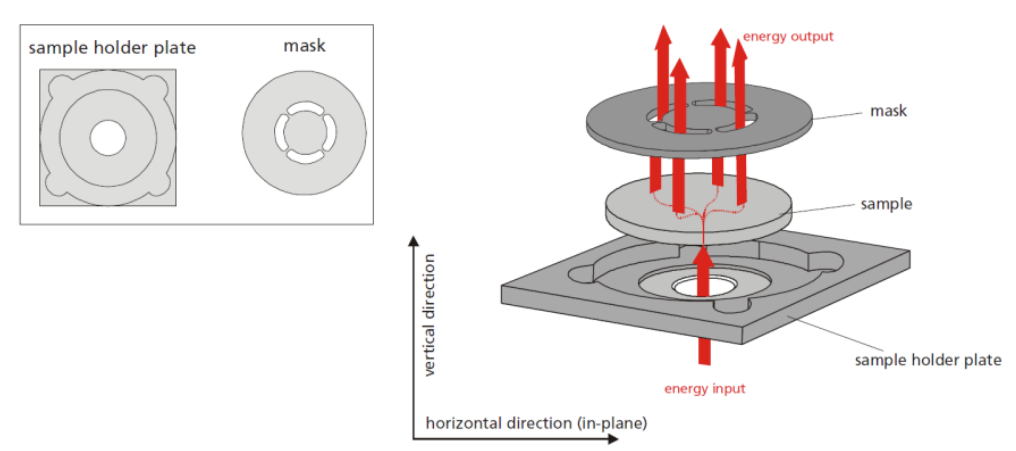

Figure 2. In-plane holder for measurements of in-plane diffusivity of anisotropic samples (Image courtesy of Netzsch)

\section{Results and Discussions}

In Figure 3 are reported the values of thermal conductivity of a sample of stretched UHMWPE (draw ratio of 200) as a function of temperature. The values at room temperature $(46 \mathrm{~W} / \mathrm{m} \mathrm{K})$ are two orders of magnitude higher than the values normally measured for bulk PE, thus confirming the dramatic effect induced by chain alignment.

The drop in thermal conductivity is observed around $70^{\circ} \mathrm{C}$ : we attribute this drop to the alpha-relaxation of polyethylene. ${ }^{7}$ 


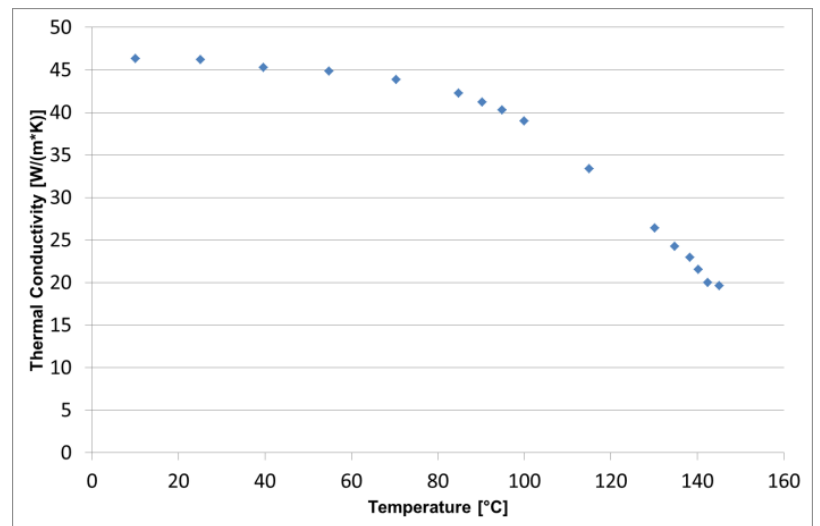

Figure 3. Thermal conductivity on a sample of UHMWPE stretched to a draw ratio of 200 as a function of temperature.

Figure 4 shows a typical plot of the pulse data fitted with the model isotropic, in-plane + heat loss

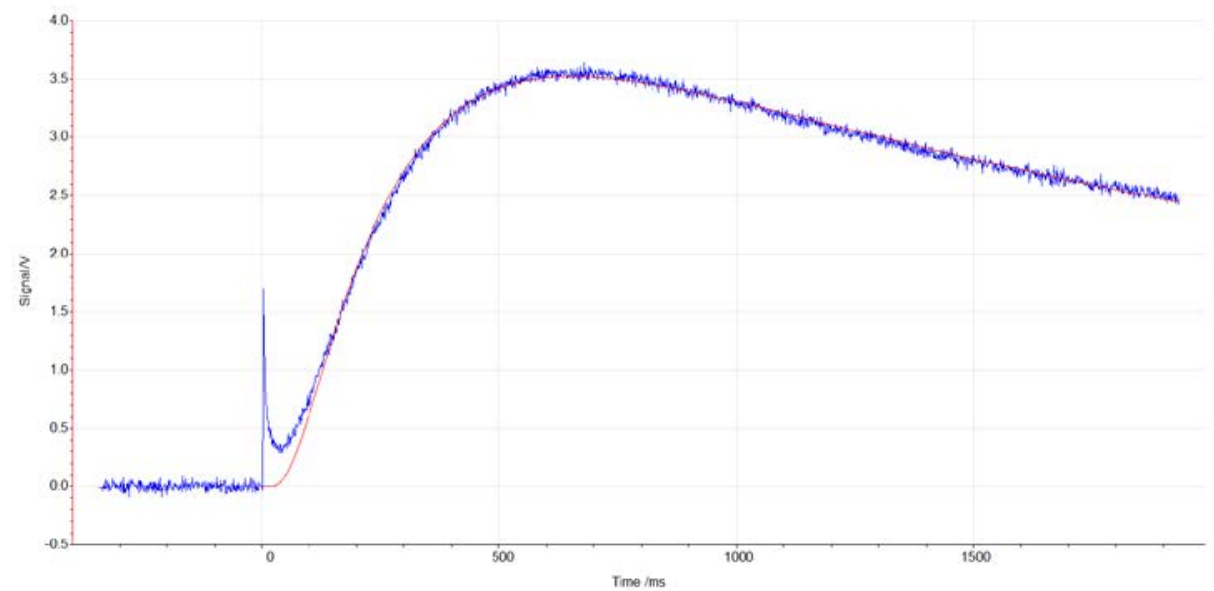

Figure 4. A typical pulse data from laser-flash in-plane measurement.

In Figure 5 are reported for comparison the values of thermal conductivities of various materials:

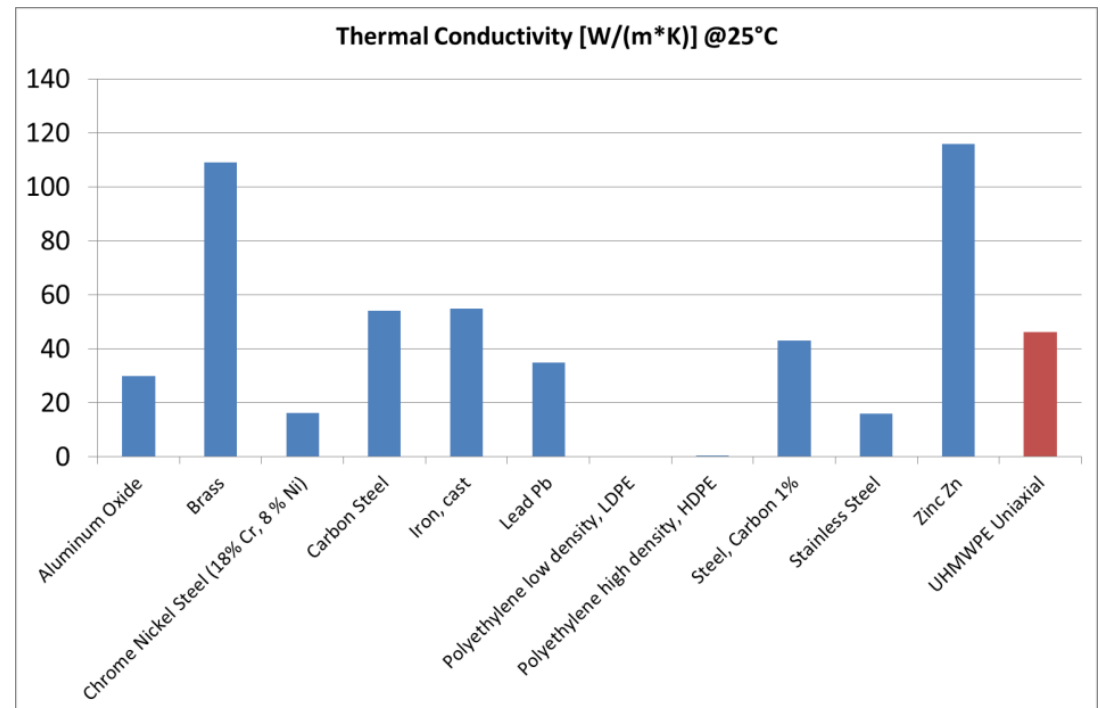

Figure 5. Thermal conductivities of various materials: the stretched UHMWPE of this study is reported in red. 


\section{Materials and methods}

\subsection{Materials}

The UHMWPE used for the analysis was synthesised according to reference 1 . The main molecular characteristics were $M_{\mathrm{w}}=5.5 \times 10^{6} \mathrm{~g} / \mathrm{mol}$, Molecular Weight Distribution $(\mathrm{MWD})=2.5$, melting temperature, $T_{\mathrm{m}}=$ $140^{\circ} \mathrm{C}$.

\subsection{Uniaxial stretching of UHMWPE}

A mould has been used to sinter samples of size $50 \times 40 \times 0.5 \mathrm{~mm}$ at $125^{\circ} \mathrm{C}$. Samples have been rolled between roll mills at $130^{\circ} \mathrm{C}$ and then marked every $0.5 \mathrm{~mm}$. Samples were stretched by using a Hounsfield tensometer at $100 \mathrm{~mm} / \mathrm{min}$ equipped with an ambient chamber set at $135^{\circ} \mathrm{C} .3$ regions of stretching could be observed with average thickness around 27um, 33um and 43um.

\subsection{Preparation of samples for Laser-flash}

Samples were cut by hand into circular-like shapes with minimum diameter of $22 \mathrm{~mm}$. Samples were placed into in-plane holders and treated with Graphite spray. Samples' heat diffusivity was measured at different temperatures and converted using Proteus Analysis 7.0; via isotropic, in-plane + heat loss method. The thermal conductivity was calculated using density $\rho=970 \mathrm{~kg} / \mathrm{m}^{3}$ and specific heat capacity of polyethylene from reference 8 .

\section{Conclusions}

The possibility to measure thermal diffusivity of highly-oriented samples of UHMWPE through laser-flash, inplane thermal analysis has been investigated. The direct measurements of thermal diffusivity can give an indirect estimation of thermal conductivity, once the bulk density and specific heat capacity of the material are known. The use of in-plane type of holders is required due to the anisotropic nature of the samples, induced by the uniaxial stretching. The high orientation of the long UHMWPE chains results in thermal conductivities superior to steel.

\section{ACKNOWLEDGEMENTS}

This project has been funded by the Engineering and Physical Science Research Council (EPSRC), grant EP/K034405/1.

\section{REFERENCES}

\footnotetext{
${ }^{1}$ Rastogi, S., Yao, Y., Ronca, S., Bos, J., van der Eem, J. Macromolecules 2011, 44, 5558.

${ }^{2}$ Ronca, S.; Forte, G.; Tjaden, H.; Rastogi, S. Ind. Eng. Chem. Res. 2015, 54, 7373.

${ }^{3}$ Choy, C.L.; Luk, W.H.; Chen, F.C. Polymer 1978, 19, 155.

${ }^{4}$ Shen, S.: Henry, A.: Tong, J.: Zheng, R.: Chen, G. Nat. Nanotechnol. 2010, 5, 251.

${ }^{5}$ Nunes dos Santos, W.; Mummery, P.; Wallwork, A. Polymer Testing 2005, $24,628$.

${ }^{6}$ Parker, W.J.; Jenkins, R.J.; Butter, C.P.; Abbot, G.L. J. Appl. Phys. 1961, 32, 1679.

${ }^{7}$ Sinnott, K.M. J. Pol. Sci. Part B 1965, 3, 945.

${ }^{8}$ Gaur, U.; Wunderlich, B. J. Phys. Chem. Ref. Data 1981, 10, 119.
} 